\title{
OPEN Experimental realization of a Fresnel hologram as a super spectral resolution optical element
}

\author{
Mei-Li Hsieh ${ }^{1,2}$, Thomas D. Ditto ${ }^{3}$, Yi-Wen Lee ${ }^{4}$, Shiuan-Huei Lin ${ }^{4}$, Heidi J. Newberg ${ }^{1}$ \& \\ Shawn-Yu Lin ${ }^{1,5 \bowtie}$
}

\begin{abstract}
A highly dispersive, diffractive optical element is designed and realized for an extremely high spectral resolution spectroscopy for exoplanet telescope application. Our design uses an annular Fresnel hologram to transform incident starlight directly into a spectrogram. The recording of the hologram is accomplished using two spherical waves of different radius of curvature. The resultant hologram consists of an annular grating structure with a gradually shrinking period as a function of increasing radius. The variable period not only could bring the incoming star-light into focus, but also exhibits a large on-axis chromatic behavior. We demonstrate a dispersion of wavelength 430-700 nm over $190 \mathrm{~mm}$ on-axis distance, leading to a super fine spectral resolution $0.0266 \mathrm{~nm}$ at wavelength $515 \mathrm{~nm}$ for a detector size of $20 \mu \mathrm{m}$.
\end{abstract}

For the past two decades, the phenomena of extreme light guiding, bending and dispersing based on the principle of diffraction has attracted much attention. Examples are sharp angle light-bending at one wavelength by a photonic crystal structure ${ }^{1}$, ultra sensitive light-dispersion in a photonic crystal structure ${ }^{2}$ and 90 -degree light-bending in a volume hologram ${ }^{3,4}$. Furthermore, diffractive optical elements (DOEs) can achieve more complex functionalities than traditional refractive optical elements, such as lenses, prisms or aspheres, could. Also, DOE is much lighter in their mass density. Hence, DOEs offer an appealing solution for the construction of next-generation, ultra-large $>20 \mathrm{~m}$ space telescope primaries ${ }^{5-10}$. However, diffractive optical elements may produce a large chromatism when illuminated with a broad-bandwidth white light source. Nonetheless, the high chromatism of a DOE may be advantageous for spectroscopic applications. As an example, a novel telescope, Dual Use Exoplanet Telescope (DUET) ${ }^{11}$, was designed to directly analyze spectra of an exoplanet with extremely high spectral resolution based on diffractive optics. DUET can simultaneously perform high resolution spectroscopy and has a low areal mass for space exploration. Figure 1 shows a schematic diagram of such an exoplanet telescope. The main component of the telescope is an annulus gossamer membrane holographic primary objective with large collection area, yet its mass and stowage allow it to be delivered on a single lifter. In the inset, we show a photo of a diffracted image of sunlight when passing through our Fresnel hologram objective.

In this paper, we show a successful design and construction of a Fresnel hologram to focus light in the transverse direction ( $\mathrm{x}-\mathrm{y}$ plane) and disperse it in a linear fashion along the optical axis ( $\mathrm{z}$-axis). Previously, a holographic optical element (HOE) has been proposed to function as lenses for optical imaging purpose and was modeled using ray tracing method ${ }^{12}$. In this study, our Fresnel hologram is designed to function not only as a focusing but also a dispersive element along an optical axis. Additionally, the computational method we used is based on wave-optics principle. While typical FZP (Fresnel Zone Plate) ${ }^{13}$ is used for beam focusing of a specific wavelength, our hologram is designed to dispersed broad wavelengths over a long distance on axis. This Fresnel hologram constitutes the primary diffractive optics in a DUET system ${ }^{10,11}$. The main function of the Fresnel hologram is to collect the collimated star-light source and focus it into the detector. When a broad-band light source passes through an annulus diffractive optic, the incident light with different wavelengths will be focused into different positions along the optical axis. It is called dispersive behavior and can be applied for high resolution spectroscopy applications. In this paper, we will focus our effort on designing the annulus Fresnel hologram, realizing this hologram and demonstrating its unusually large chromatic behavior.

\footnotetext{
${ }^{1}$ The Department of Physics, Applied Physics and Astronomy, Rensselaer Polytechnic Institute, Troy, NY 12180, USA. 'Department of Photonics, National Yang Ming Chiao Tung University, Hsinchu, Taiwan. ${ }^{3} 3$ DeWitt LLC, P.O. Box 10, Ancramdale, NY 12503-0010, USA. ${ }^{2}$ Department of Electrophysics, National Yang Ming Chiao Tung University, Hsinchu, Taiwan. ${ }^{5}$ College of Photonics, National Yang Ming Chiao Tung University, Hsinchu, Taiwan. ${ }^{\varpi}$ email: sylin@rpi.edu
} 


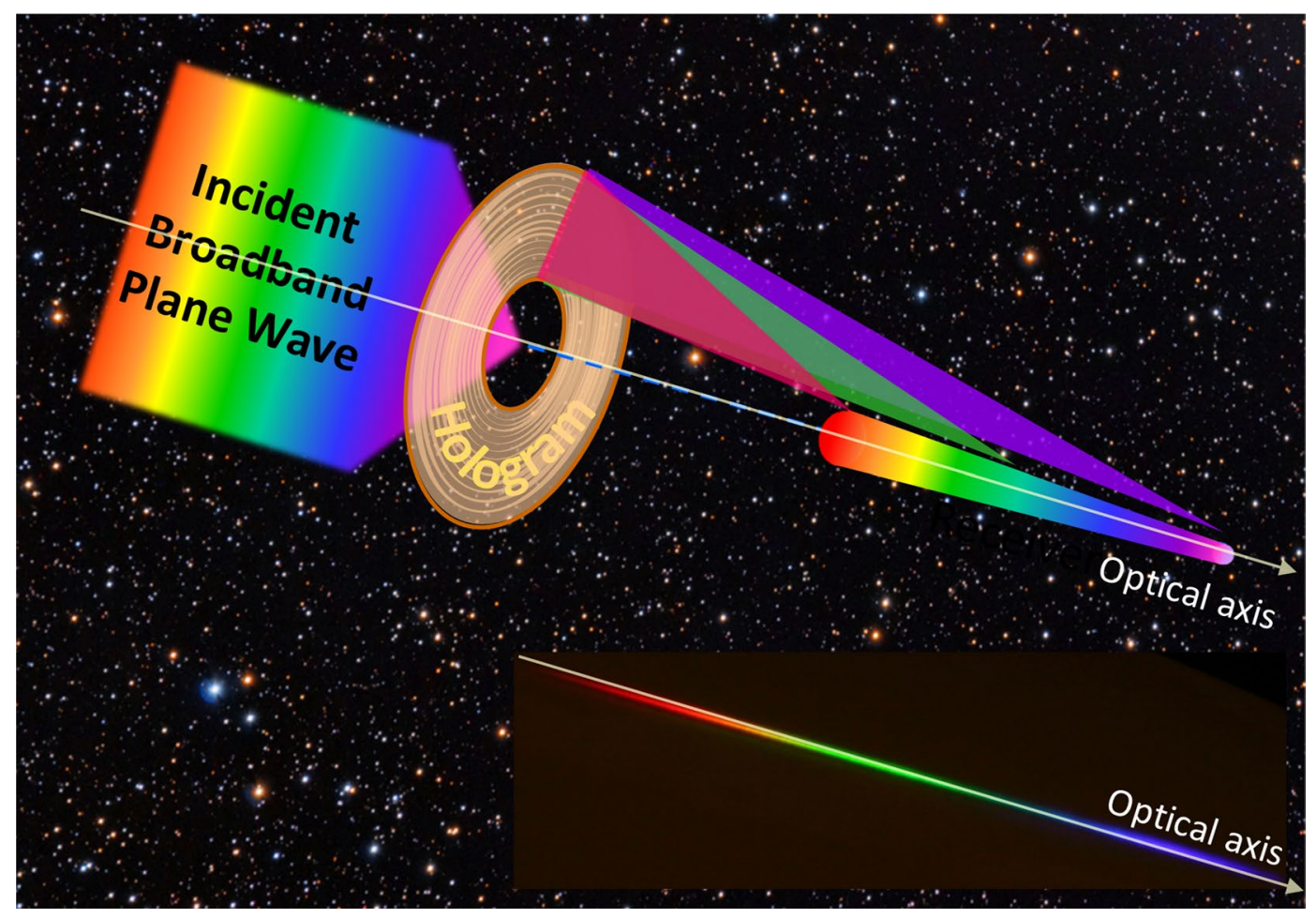

Figure 1. A schematic diagram of the dual use exoplanet telescope (DUET) based on a Fresnel hologram. (Inset) The photo shows a diffracted image of sunlight when passing through a Fresnel hologram.

\section{Design and simulation results of the Fresnel hologram}

Our Fresnel hologram is based on the interference of two spherical waves, emerging from point sources $U_{\mathrm{R}}$ (Reference) and $U_{\mathrm{O}}$ (Object), on a hologram film located at $z=0$ plane. Note that a typical Fresnel zone plate is produced using a plane-wave and a spherical wave, which may be viewed as a special case of our two sphericalwaves configuration. Figure 2a shows a schematic diagram of the two-wave recording geometry. The reconstruction of the hologram is achieved by placing another point source $U_{\mathrm{P}}$ at the same side of $U_{\mathrm{R}}$ and $U_{\mathrm{O}}(z<0)$ and then searching for point image formation at the other side of the hologram, i.e. $z>0$. The recording wavelength, $\lambda_{\mathrm{i}}$, of $U_{\mathrm{R}}$ and $U_{\mathrm{O}}$ may be different from the reading one of $U_{\mathrm{p}} \lambda$. When the propagating waves from $U_{\mathrm{R}}, U_{\mathrm{O}}$ and $U_{\mathrm{P}}$ satisfy the paraxial approximation ${ }^{14}$, formation of a point image can occur near or on the optical axis. Also, noted that the focal length of the point image is expected to be $\lambda$-dependent.

In this paper, we consider two special conditions: (1) both $U_{\mathrm{R}}$ and $U_{\mathrm{O}}$ are placed on the $z$-axis, leading to an on-axis hologram with cylindrical symmetry and annular shape of the interference pattern; (2) $U_{\mathrm{P}}$ is placed quasi-infinitely away from the hologram, so it has a planar wave-front. Under this configuration, our hologram can image (or concentrate) a collimated, single $\lambda$ beam onto a point on the axis. It can also image a broadband, collimated beam onto a series of points along the axis. Traditionally, when a broadband radiation of a given intensity is dispersed in space, it gains angular spread of $\lambda$ but at the expense of losing its intensity flux. On the contrary, for our Fresnel hologram, a broadband radiation is dispersed along the optical axis but each wavelength of light is focus on its own focal point. This is an important feature of our hologram, leading to both super $\lambda$-resolution and super sensitive detection for an exoplanet telescope.

We now present a brief derivation of the theoretical framework of image formation. A more detail account of it is discussed in the Method section. From Fig. $2 \mathrm{a}$, the reference wave $U_{\mathrm{R}}$ is on $\left(x_{\mathrm{R}}, y_{\mathrm{R}}, z_{\mathrm{R}}\right)$ and the objective wave $U_{\mathrm{O}}$ on $\left(x_{\mathrm{O}}, y_{\mathrm{O}}, z_{\mathrm{O}}\right)$. The two recording waves propagate along the optical axis and interfere at $z=0$ plate. The intensity distribution of the interference pattern of the two spherical waves can be written as:

$$
I(x, y, z)=\left|U_{R}+U_{O}\right|^{2} \approx \mid A_{\mathrm{R}} \frac{e^{i k \sqrt{\left(x-x_{R}\right)^{2}+\left(y-y_{R}\right)^{2}+\left(z-z_{R}\right)^{2}}}}{\left|z_{R}\right|}+A_{\mathrm{O}} \frac{\left.e^{i k \sqrt{\left(x-x_{o}\right)^{2}+\left(y-y_{o}\right)^{2}+\left(z-z_{O}\right)^{2}}}\right|^{2}}{\left|z_{O}\right|}
$$

Here, $A_{\mathrm{R}}$ and $A_{\mathrm{O}}$ are the amplitude of the reference and objective beams, respectively. $k$ is the recording beam wave vector, $k=2 \pi / \lambda$ and $\lambda_{\mathrm{i}}$ is its wavelength. Under the Paraxial approximation. $U_{\mathrm{R}}$ and $U_{\mathrm{O}}$ may be re-written as : 

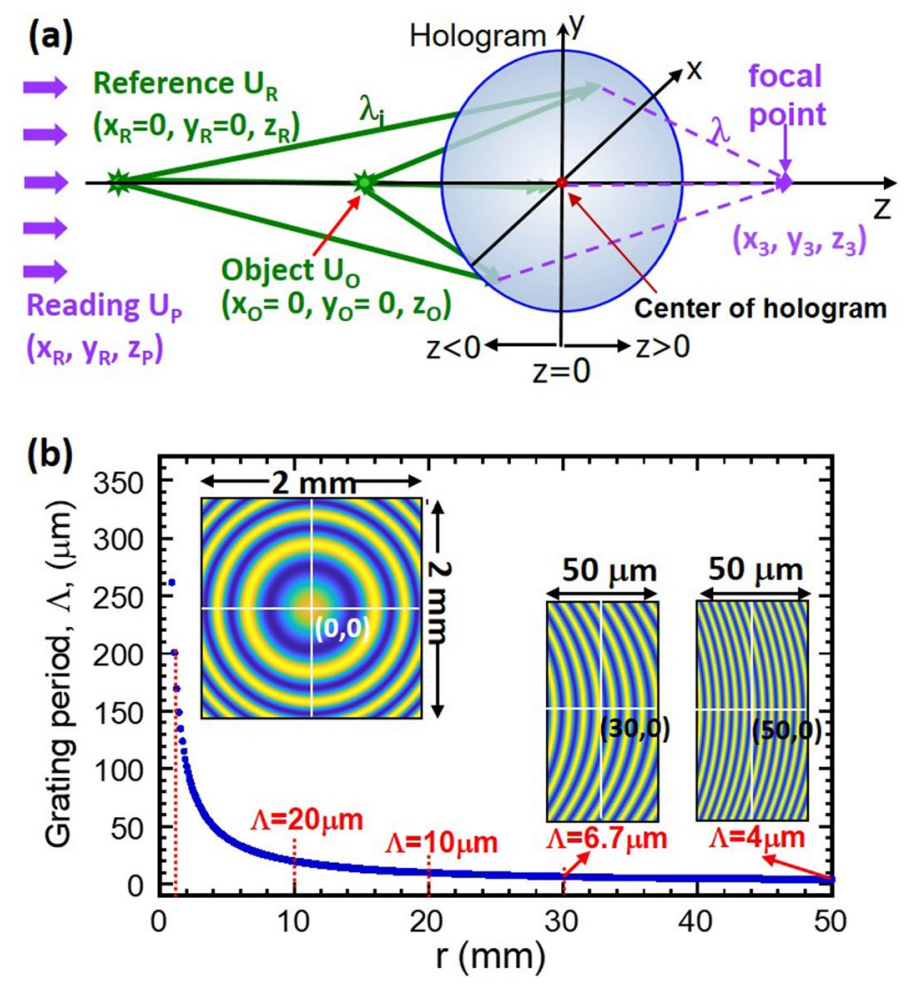

Figure 2. Experimental recording of the Fresnel hologram. (a) A schematic of the recording optical system using a diode-pumped-solid-state laser at $\lambda=515 \mathrm{~nm}$. The reference and object are both point sources, emitting spherical waves for hologram recording. (b) A plot of the resulting grating pitch v.s. radial distance $(r)$ away from the center of the hologram. (Inset) Images of intensity distribution of the interference pattern at three different positions of the hologram, i.e. $(x, y)=(0,0),(30 \mathrm{~mm}, 0)$ and $(50 \mathrm{~mm}, 0)$.

$$
\begin{aligned}
U_{R} & =\left(A_{R} / z_{R}\right) e^{i k \sqrt{\left(x-x_{R}\right)^{2}+\left(y-y_{R}\right)^{2}+\left(z-z_{R}\right)^{2}}} \approx\left(A_{R} / z_{R}\right) e^{i k \phi_{R}} \\
U_{O} & =\left(A_{O} / z_{O}\right) e^{i k \sqrt{\left(x-x_{O}\right)^{2}+\left(y-y_{O}\right)^{2}+\left(z-z_{O}\right)^{2}}} \approx\left(A_{O} / z_{O}\right) e^{i k \phi_{O}} \\
\phi_{R} & \equiv-\frac{\pi}{\lambda_{i} z_{R}}\left(x^{2}+y^{2}-2 x x_{R}-2 y y_{R}\right) \\
\phi_{O} & \equiv-\frac{\pi}{\lambda_{i} z_{O}}\left(x^{2}+y^{2}-2 x x_{O}-2 y y_{O}\right)
\end{aligned}
$$

To simplify Eq. (1) and also to achieve the maximum contrast of the interference pattern, the amplitude of the reference and objective beams are set to $A_{\mathrm{R}} /\left|z_{\mathrm{R}}\right|=A_{\mathrm{O}} /\left|z_{\mathrm{O}}\right|=1$. Thus, the intensity distribution of the interference pattern becomes:

$$
I(x, y, z)=\left|e^{i k \sqrt{x^{2}+y^{2}+\left(z-z_{R}\right)^{2}}}+e^{i k \sqrt{x^{2}+y^{2}+\left(z-z_{O}\right)^{2}}}\right|^{2}
$$

To illustrate the annular feature of the hologram, we compute the intensity distribution of the interference pattern using Eq. (3). The results are shown in the inset of Fig. $2 \mathrm{~b}$. Here, $\lambda_{\mathrm{i}}=515 \mathrm{~nm}$ and the two spherical waves are located at $z_{\mathrm{R}}=-72 \mathrm{~cm}$ and $z_{\mathrm{O}}=-25.2 \mathrm{~cm}$, respectively. The interference pattern at the origin $(x, y)=(0,0)$ is shown on the upper left over an area $2 \times 2 \mathrm{~mm}^{2}$. The blue color represents the minimum intensity and the yellow the maximum intensity. It exhibits an annular-like grating structure, consistent with the axial symmetry of the recording and reading configuration. Here the radius of first blue circle is $380 \mu \mathrm{m}$ and the grating pitch between the first and second blue circle is $\Lambda=260 \mu \mathrm{m}$. Additionally, the interference patterns at $(x, y)=(30 \mathrm{~mm}$, $0)$ and $(50 \mathrm{~mm}, 0)$ are shown on the lower right corner over an area $50 \times 100 \mu \mathrm{m}^{2}$. They both display a shorter grating pitch. The grating pitch is reduced to $\Lambda=6.7 \mu \mathrm{m}$ at $(30 \mathrm{~mm}, 0)$ and further reduced to $\Lambda=4 \mu \mathrm{m}$ at $(50 \mathrm{~mm}, 0)$. Theoretically, the grating pitch of the intensity distribution pattern is a function of the radial position $r=\left(x^{2}+y^{2}\right)^{1 / 2}$. Figure $2 \mathrm{~b}$ plots the grating pitch as a function $r$ as the blue color curve. The grating pitch decreases rapidly as $r$ is increased. For instance, grating pitch decreases from $\Lambda=201,20,6.7$ to $4 \mu \mathrm{m}$ as the radius is increased from $r=1,10,30$ to $50 \mathrm{~mm}$, respectively. Note that the decreasing $\Lambda$ from the origin to the outer regions serves to bring the incoming plane wave to focus much like the traditional Fresnel zone plate ${ }^{13}$. This provides for a mechanism for the hologram to strongly concentrate light to a "point" bounded only by the diffraction limit. 
The focal-point of our hologram, i.e. $\left(x_{3}, y_{3}, z_{3}\right)$, may be derived analytically as well. Again, its full derivation is discussed in the Method section. Briefly, the derivation principle is to match the phase factor from the object $\left(\phi_{\mathrm{O}}\right)$ and recording beam $\phi_{R}$ to the phase factor of the diffracted beam $\phi_{3}$. As a result, the focal point $\left(x_{3}, y_{3}, z_{3}\right)$ of the image is a function of $z_{\mathrm{R}}, z_{\mathrm{O}}, \lambda, \lambda_{i}$, and given by:

$$
\begin{aligned}
& x_{3}=y_{3}=0 \\
& \frac{1}{z_{3}}=\left(\frac{1}{z_{R}}-\frac{1}{z_{O}}\right) \frac{\lambda}{\lambda_{i}} \quad \text { or } \quad z_{3} \lambda=\left(\frac{1}{z_{R}}-\frac{1}{z_{O}}\right)^{-1} \lambda_{i}
\end{aligned}
$$

This derivation shows that, for our hologram design, the image is located on-axis $\left(x_{3}=y_{3}=0\right)$ and the product of $\left(z_{3} \lambda\right)$ is a constant. In our experimental case, when $z_{\mathrm{R}}=-72 \mathrm{~cm}, z_{\mathrm{O}}=-25.2 \mathrm{~cm}, \lambda_{\mathrm{i}}=515 \mathrm{~nm}$, $\left(z_{3} \lambda\right)=19,966 \mathrm{~cm}-\mathrm{nm}$. Note that $z_{3}$ is a positive number. This means that the diffracted output is focused onto the $z_{3}$ position forming a real image. On contrary, if $z_{3}$ is a negative number, the diffracted output becomes a divergent wave that originates from $-\left|z_{3}\right|$ position. Hence, both the recording parameters, $z_{\mathrm{R}}$ and $z_{\mathrm{O}}$, provides for a new design degree-of-freedom for producing either a convergent or divergent diffracted beam. The combinational use of two spherical waves also allow us to design a large value of $z_{3}$ (when $z_{\mathrm{R}} \cong z_{\mathrm{O}}$ ), and thus can produce a highly dispersive optical element. The position $z_{3}$ of the diffracted spherical wave, measured from the hologram plane, is called the focal-length of the hologram.

Because $z_{3}$ is inversely proportional to the reading $\lambda$, our hologram has a significant chromatic dispersion property when illuminated with a broadband light source. When $\lambda=\lambda_{\mathrm{i}}=515 \mathrm{~nm}, z_{30}=38.8 \mathrm{~cm}$ and is the reference focal-length of the image. When $\lambda \neq \lambda_{i}$, the diffracted wave is focused onto a different position along $z$-axis such that $z_{3} \neq z_{30}$. For instance, when $\lambda$ is varied from $\lambda=400 \mathrm{~nm}$ to $700 \mathrm{~nm}$, the corresponding focal length is shifted from $z_{3}=49.9 \mathrm{~cm}$ to $28.5 \mathrm{~cm}$. This result shows that the hologram can achieve a spectral dispersion of $400 \mathrm{~nm}$ to $700 \mathrm{~nm}$ wavelength over an on-axis distance of $21.4 \mathrm{~cm}$. An important feature of our hologram is its ability to achieve a long tuning length for a super high spectral resolution. It originates from an unusual on-axis dispersive behavior of the Fresnel hologram.

To independently confirm the chromatic dispersion of our hologram, we use commercially available software, Zemax ${ }^{\infty 15}$, to simulate the performance of our hologram. The simulated result is illustrated in Fig. 3. In this simulation, the hologram has a circular size with a diameter $D=10 \mathrm{~cm}$. It is modeled in accordance with our recording parameters, i.e. the two point sources are at $z_{\mathrm{R}}=-72 \mathrm{~cm}$ and $z_{\mathrm{O}}=-25.2 \mathrm{~cm}$ and the recording $\lambda_{i}=515 \mathrm{~nm}$. Also, its grating period varies as a function of $\mathrm{r}$ as shown in Fig. $2 \mathrm{~b}$. The illuminating light is chosen to be collimated and contains three different colors, i.e., $\lambda=600 \mathrm{~nm}$ (red), $515 \mathrm{~nm}$ (green) and $400 \mathrm{~nm}$ (blue). The result shows that the three colors of light are been focused onto three different spots along the $z$-axis. We obtain $z_{3}(\lambda=600 \mathrm{~nm})=333 \mathrm{~mm}, z_{3}(\lambda=515 \mathrm{~nm})=389 \mathrm{~mm}$, and $z_{3}(\lambda=400 \mathrm{~nm})=500 \mathrm{~mm}$. We found that the shift of the focal length versus $\lambda$ is consistent with that predicted from Eq. (4). This result numerically demonstrates the unusually large chromatic dispersion produced by our hologram. Figure 3 also plots the intensity distribution of the three wavelengths at their respective focal plane and along the $x$-axis. The spatial intensity distributions for all three colors are sharp. We found the diameter of the focus points to be less than $5 \mu \mathrm{m}$. Although, we note that Zemax ${ }^{\circledast}$ is based on ray tracing method and the minimum focus spot should be set by the diffraction limit. Nonetheless, the intensity distribution plots illustrate the unusual light concentration capability of our hologram. This simulation shows that our hologram can achieve strong concentration of a single color and also on-axis stretching of multi-colors all at the same time.

\section{Experimental discussion}

We have established an optical system to realize the Fresnel hologram and experimentally demonstrate the chromatic behavior of the hologram. Figure 4a shows a schematic diagram of the optical setup system. The laser we used for hologram recording is a collimated, diode-pumped-solid-state (DPSS) laser with a single longitudinal mode output. It emits light at $\lambda=515 \mathrm{~nm}$ and has a long coherent length of about one-meter. The laser light is first split into two beams, where one is the reference and the other the object beam. The half-wave $(\lambda / 2)$ plate is used to adjust the relative intensity of the reference and objective beams. Both beams are focused through $40 \mathrm{X}$ objective lenses and then passed through pinholes of $15 \mu \mathrm{m}$ diameter. This procedure produces point light source and, therefore, the desirable propagating spherical waves. The pinholes for the reference and object beams are located at $z_{\mathrm{R}}=-72 \mathrm{~cm}$ and $z_{\mathrm{O}}=-25.2 \mathrm{~cm}$ away from the hologram plane, respectively. In order to record an onaxis hologram, an additional one-inch mirror is used to guide and align the object beam such that it propagates along the same optical axis as the reference beam. Finally, a Litiholo holographic film of 4 " $\times 5$ " size is placed at the desired exposure position to record the hologram. Figure $4 \mathrm{~b}$ shows a photo of the optical recording system for our Fresnel hologram. Note the size of a realizable hologram is limited by the power and finite coherent length of a laser. Using our current optical setup system, the hologram might be scaled up to about one-meter in diameter.

Once the hologram is recorded, a high resolution microscope is used to verify if the resultant interference pattern follows the design prediction shown in Fig. 2b. Figure 5a shows a schematic drawing of the hologram and coordinates where microscope images were taken. Figure 5 b shows a photo of the $4 " \times 5$ " hologram film after been exposed to $\sim 40 \mathrm{~mJ} / \mathrm{cm}^{2}$ recording energy. Figure $5 \mathrm{c}-\mathrm{e}$ show microscope images of the hologram at point $r_{1}=(0,25 \mathrm{~mm}), r_{2}=(40 \mathrm{~mm}, 0)$ and $r_{3}=(50 \mathrm{~mm}, 0)$, respectively. In all three images, clear grating structures were observed that illustrate a successful interference of the reference and object beams. The grating period is systematically reduced from $\Lambda \sim 9.1,5.6$ to $4.1 \mu \mathrm{m}$ as the coordinates radius is increased from $r=25,40$ to $50 \mathrm{~mm}$, respectively. The decreasing trend of $\Lambda$ toward outward region of the hologram is consistent with the trend predicted in Fig. 2b. It is noted that the theoretical simulation predicts a grating period of $\Lambda \sim 8.0$ and $4.0 \mu \mathrm{m}$ at $r=25$ and $50 \mathrm{~mm}$, respectively. There are two reasons that might cause a slight discrepancy between the observed 


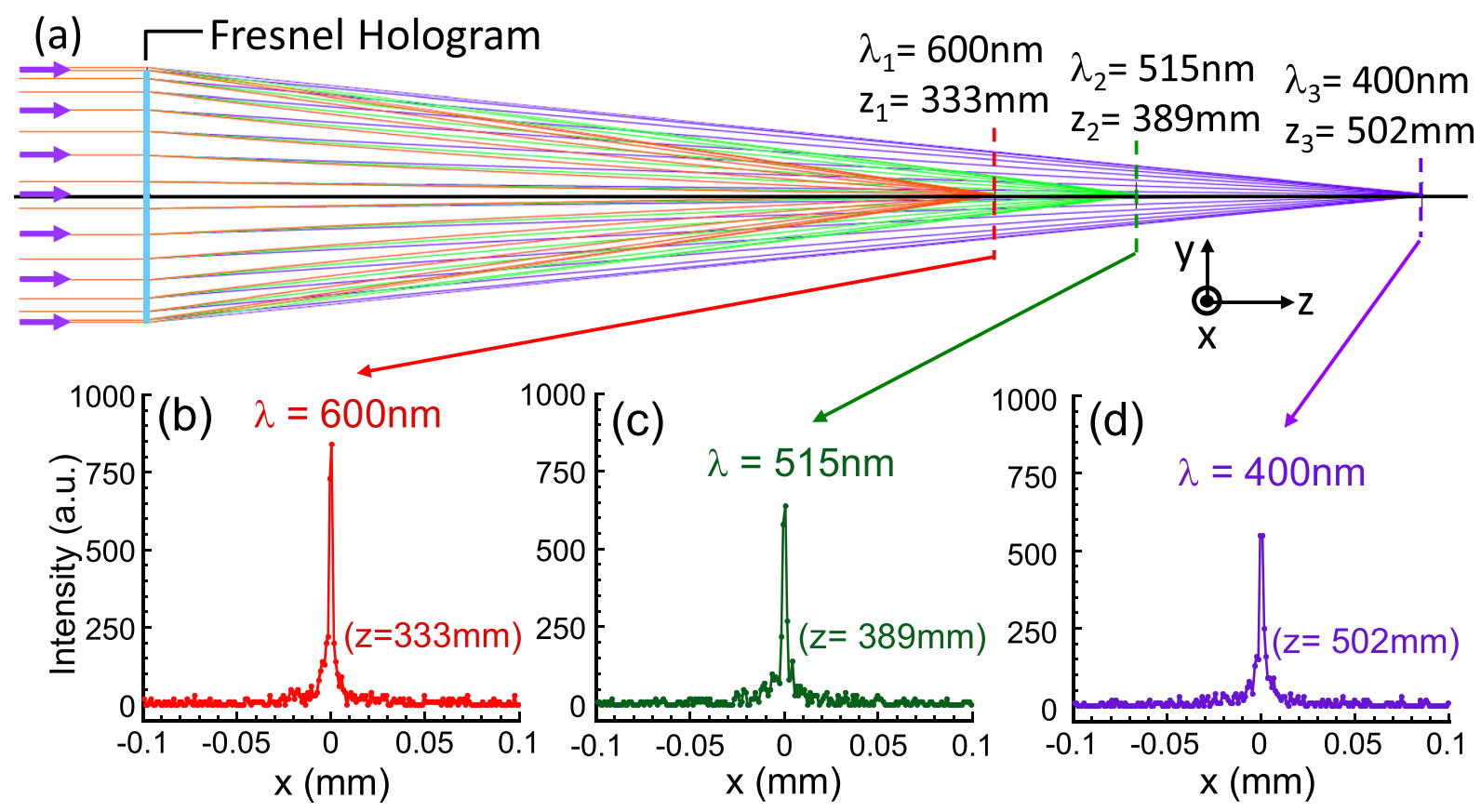

Figure 3. A simulation result of the reconstructed image at three different wavelength of light, $\lambda_{1}=600$, $\lambda_{2}=515$ and $\lambda_{3}=400 \mathrm{~nm}$. (a) The effect of light focusing and dispersion by a Fresnel Hologram simulated by the ZEMAX software. (b-d) are the simulated intensity distribution of light versus $\mathrm{x}$-axis of three different ls at their respective focal planes, $z_{1}=333, z_{2}=389$ and $z_{3}=520 \mathrm{~mm}$, respectively.

and predicted grating period. One is due to a non-ideal optical alignment between the two recording beams. For instance, from our calculation, if the two beams are not perfectly aligned along the same direction and off by $0.1^{\circ}$, it will result in a deviation of grating period by $\sim 1.1 \mu \mathrm{m}$ at $r=25 \mathrm{~mm}$. Conversely, it may be stated that the upper bound of our alignment error is within $0.2^{\circ}$. The other is a possible measurement error of finding the exact central position of the almost transparent hologram under the microscope. Nonetheless, the observation of clear grating structures and the agreement of grating periods between experiment and theory throughout the hologram film indicate that we have successfully recorded a Fresnel hologram.

The recorded hologram is expected to function simultaneously as a chromatic and a focusing element. Figure 6 experimentally illustrates the chromatic behavior of the hologram. A white LED light (covering $\lambda=450-650 \mathrm{~nm}$ spectral range) is used to illuminate the hologram. The LED light serves as a point source, which is expanded and collimated using a Cassegrain reflecting telescope ${ }^{16}$ in a reverse input configuration and normally incident onto the hologram. The transmitted wave is then diffracted and imaged onto different positions on the $z$-axis in accordance to their specific $\lambda$. Figure 6 a shows a photo of the reconstructed image of the hologram. Firstly, the white LED light is well focused and dispersed along the optical axis, where a ruler is carefully placed near the axis. The finite spread of light along the $y$-direction is due to a slight mis-alignment of the ruler to the $\mathrm{z}$-axis and also the scattering of light by the ruler. The actual spot size is much smaller and is defined by the diffraction limit of the hologram size, see Fig. 3b. Secondly, the white LED light is dispersed into a series of vivid colors, ranging from red, orange, yellow, green, blue and purple. The observation of bright color (i.e. strong intensity) for a dispersed light is in sharp contrast to those of typical dispersive elements, such as a prism, where light is dispersed, spread in all three dimensions and its intensity flux greatly weakened. Finally, consistent with the prediction of Eq. (8), the shorter wavelength $(\lambda)$ light has a longer focal point $\left(z_{3}\right)$.

While the concentration capability of our hologram is characterized by a tight spatial extend of the focused spot in the transverse plane $(x-y)$ shown in Fig. 3b, its chromatic behavior is quantified by a long spatial extend along the longitudinal axis $(z$-axis). Here we perform a spectroscopic study of the spectral resolution of the focused light at a series of focal points along the $z$-axis. A fiber coupled optical spectrometer (Ocean Optics USB4000) with a fiber core diameter of $d=400 \mu \mathrm{m}$ is utilized to measure the spectrum. The fiber, shown in the far left of Fig. 6a, is to be translated along the $z$-axis to perform spectroscopic measurement. Figure $6 \mathrm{~b}$ shows a series of measured spectra taken at a range of $z$-position from $z=28 \mathrm{~cm}$ to $47 \mathrm{~cm}$. Firstly, the measured spectra show stronger intensities at $\lambda \sim 455 \mathrm{~nm}$ (blue color) and $\lambda \sim 550 \mathrm{~nm}$ (yellow) region. This is due to spectral distribution of the primary and secondary emission of the LED light used in our testing ${ }^{17}$. Secondly, the measured spectra displayed well-defined peak wavelength, which are shifted systematically toward longer- $\lambda$ for shorter $z$. The full-width-half-maximum (FWHM) of the individual peak is $\Delta \lambda_{\mathrm{FWHM}} \sim 7.5 \mathrm{~nm}$ at $\lambda \sim 450 \mathrm{~nm}$ and increased by about two times to $\Delta \lambda_{\text {FWHM }} \sim 15 \mathrm{~nm}$ at $\lambda \sim 650 \mathrm{~nm}$. The relatively large FWHM is mainly due to the finite size of the fiber core diameter $d=400 \mu \mathrm{m}$. It does not represent the intrinsic spectral resolution of the hologram, which is to be addressed in a following paragraph.

Thirdly, the measured peak is shifted from $\lambda=440 \mathrm{~nm}$ to $705 \mathrm{~nm}$ as the focal length is decreased from $z=470 \mathrm{~mm}$ to $280 \mathrm{~mm}$. So, white light of $\Delta \lambda=265 \mathrm{~nm}$ bandwidth is well dispersed into a spatial extend of 

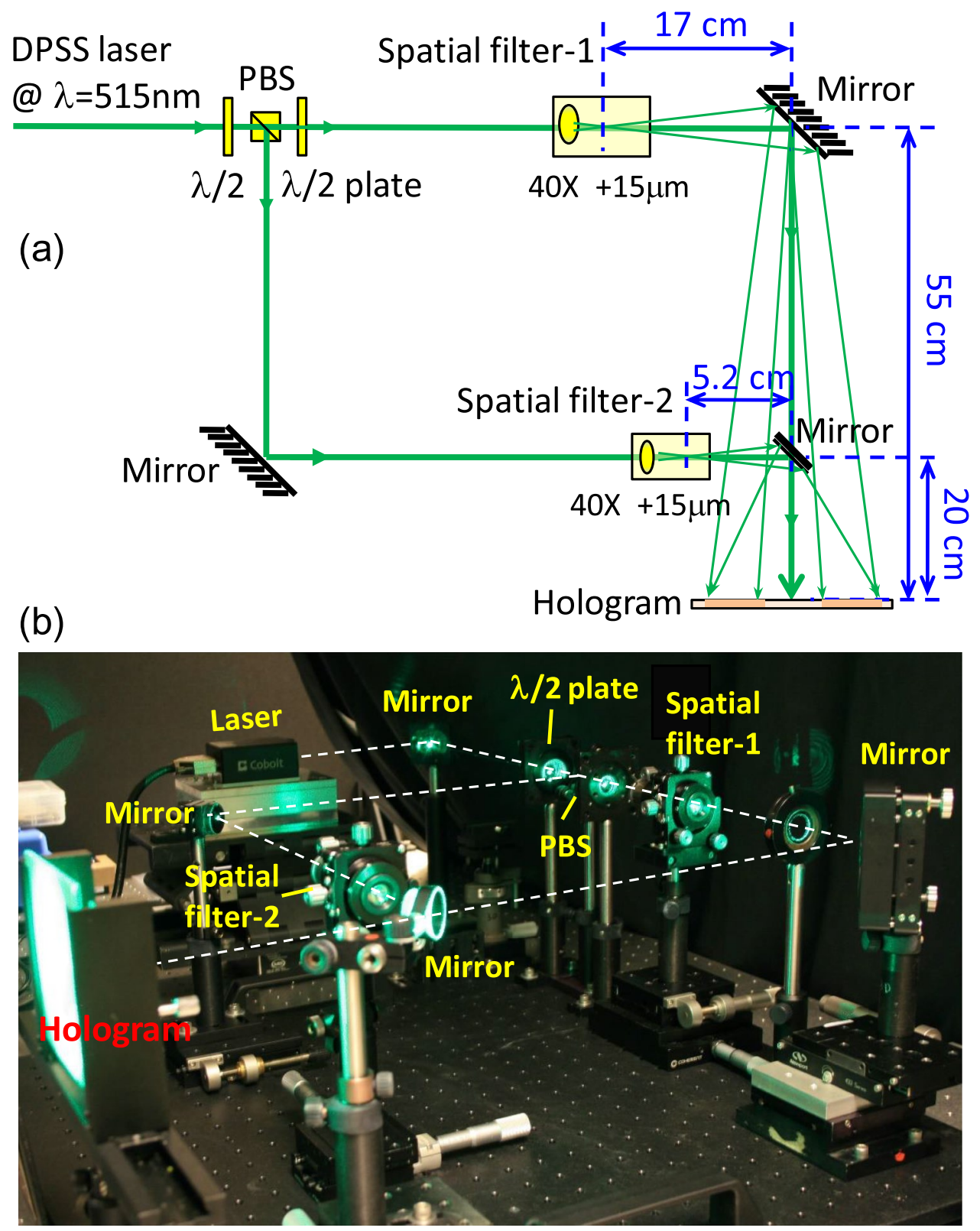

Figure 4. (a) A schematic diagram of the optical recording system for Fresnel Hologram. (b) A photo of the recording setup using a $\lambda=515 \mathrm{~nm}$ laser. The optical path of light is indicated by white dashed lines.

$\Delta z=190 \mathrm{~mm}$. Figure $7 \mathrm{~b}$ summarizes the measured (red dots) and predicted (blue curve) focal length as a function of $\lambda$. The measured focal length shows a decreasing trend as $\lambda$ is increased. Recall from Eq. (4) that $z_{3} \lambda=z_{30} \lambda_{i}$, or

$$
z_{3}(\lambda)=\left(\frac{z_{30}}{\lambda}\right) \lambda_{i}
$$

where $z_{30}=\left(1 / z_{R}-1 / z_{0}\right)^{-1}$. So, from Eq. (5), the chromatic behavior, $z_{3}(\lambda)$, of our hologram is characterized by the pre-factor " $z_{30}$ ". When " $z_{30}$ " is increased by recording design, the incoming starlight will be more dispersed and the hologram is more suited for achieving super-resolution purpose. When " $z_{30}$ " is reduced, the diffracted $\lambda$ of the incoming starlight is more compressed (focused) along the $z$-axis and the hologram is more suited for high sensitivity application. Returning to experimental data, when the reading- $\lambda$ equals the recording $\lambda_{\mathrm{i}}=515 \mathrm{~nm}$, $z_{3}=z_{30}=388 \mathrm{~mm}$ (the reference). The measured focal length value is $z_{30}=385 \mathrm{~mm}$, which is in good agreement with the predicted result. Note also that $z_{3}$ should follow the $(1 / \lambda)$ dependence. And, indeed, our measured focal length accurately reproduces the predicted values (the blue curve).

In the following, we discuss spectral resolution and starlight detection sensitivity of our Fresnel hologram. The spectral resolution $\Delta \lambda$ of our hologram is determined by the detection length $\Delta z$ of a detector of choice along the z-axis. Let us assume that $\Delta z<<z_{30}=388 \mathrm{~mm}$ and rewrite Eq. (5) in a differential form, we find: $\Delta \lambda \cong-\left(\frac{\Delta z}{z_{30}}\right)\left[\frac{\lambda^{2}}{\lambda_{i}}\right]$. The formula indicates that the spectral resolution depends on the intrinsic property of the 
(a)

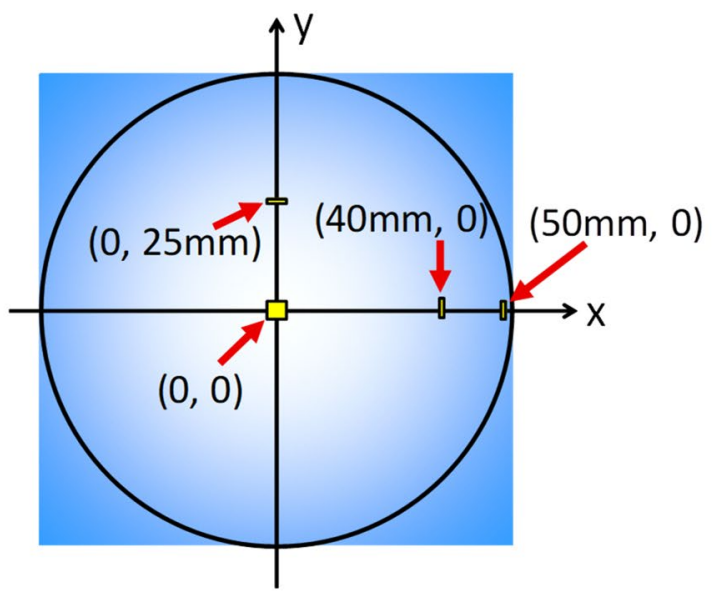

(b)

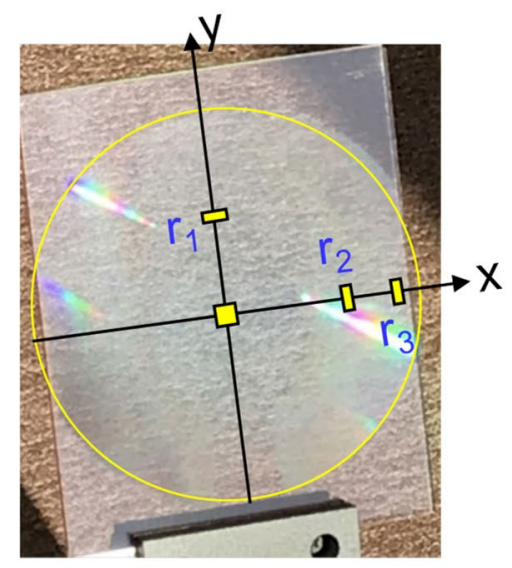

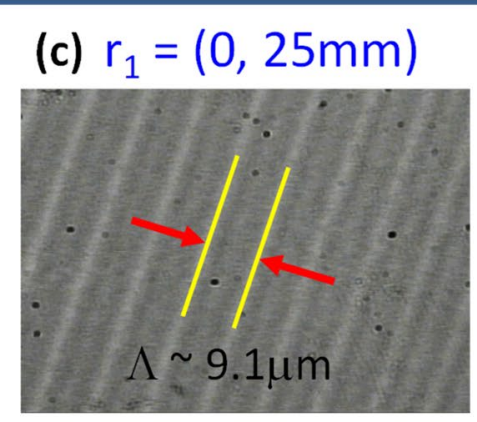

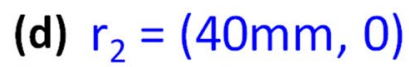

(e) $r_{3}=(50 \mathrm{~mm}, 0)$
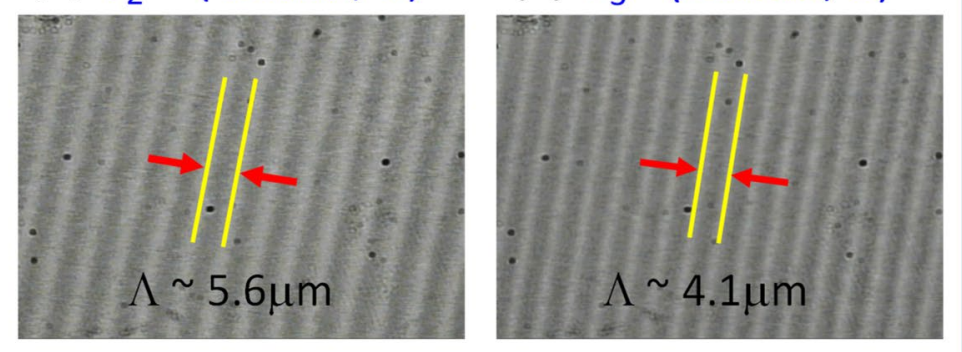

Figure 5. (a) A schematic of the hologram coordinate at $\mathrm{z}=0$ plane, where both the reference and object point sources are positioned along the $\mathrm{z}$-axis. (b) A photo of the recorded $4 \times 5 \mathrm{inch}^{2}$ Fresnel hologram on a glass plate. (c-e) Optical images of the recorded grating structure taken at different points, $r_{1}, r_{2}$ and $r_{3}$, on the Fresnel hologram. The grating pitch $\Lambda$ becomes denser as the observation point is further away from the center.

hologram $\left(\lambda_{i} z_{30}\right)$, the detection length $\Delta z$, as well as $\lambda^{2}$. This explains our earlier observation in Fig. $6 \mathrm{~b}$ that the spectral linewidth $\Delta \lambda_{\text {FWHM }}$ is wider for longer- $\lambda$ peaks. Also, when $\lambda$ is increased from 450 to $650 \mathrm{~nm}$, the theory predicts a $(650 / 450)^{2} \sim 2 \times$ increase of linewidth which agrees with the observation shown in Fig. $5 \mathrm{~b}$. As a specific example, when $\lambda=\lambda_{\mathrm{i}}=515 \mathrm{~nm}$ and $z_{30}=388 \mathrm{~mm}$, we have $\Delta \lambda \cong-\Delta z \times\left(\lambda_{i} / z_{30}\right)=-\Delta z \times 1.33 \times 10^{-3} \mathrm{~nm} /$ $\mu \mathrm{m}$. When $\Delta z=50 \mu \mathrm{m}, 5 \mu \mathrm{m}$, and $0.5 \mu \mathrm{m}$, the spectral resolution of the hologram is $|\Delta \lambda|=0.0665,0.00665$ and $0.000665 \mathrm{~nm}$, respectively. And, the corresponding spectral resolvance $(\lambda / \Delta \lambda)$ is $7.74 \times 10^{3}, 7.74 \times 10^{4}$, and $7.74 \times 10^{5}$, respectively. A resolvance of 7740 to 77,400 is in the medium to high resolution range and is a good range for determining stellar properties and radial velocities of stars in the Milky Way galaxy. A resolvance of 774,000 is in the super-resolution range for the detection of exoplanets by the radial velocity method. Noted that, to extract a selective bandwidth of light over a length $\Delta z$, a secondary optics can be used to diffract light away from the z-axis for an easy and full spectral analysis. A schematic of the approach by a diffracted optical element is illustrated in the inset of Fig. 7a.

Alternatively, a fiber or detector may be placed facing the $z$ to collect starlight, such as shown in the photo of Fig. 6a. In this case, the minimum detector size $d$ is set by the diameter of the Airy disk ${ }^{13}$ of our Fresnel hologram, i.e., $d \geq d_{\text {Airy. }}$. For our Fresnel hologram, its diameter is $D=10 \mathrm{~cm}$ and the corresponding diameter of Airy disk is $d_{\text {Airy }}=2.44 \times \frac{\lambda z_{3}}{D}=2.44 \times \frac{\lambda_{i} z_{30}}{D}=4.8 \mu \mathrm{m}$. Interestingly, since $d_{\text {Airy }}$ depends on intrinsic parameters such as $\lambda_{i}$ and $z_{30}$ and $D$, its value remains the same for all wavelengths or it is $\Delta \lambda$-independent. Additionally, if we increase the diameter of our hologram to $D^{\prime}=1 \mathrm{~m}$, the corresponding Airy disk is reduced to $d_{\text {Airy }}^{\prime}=2.44 \times \frac{\lambda_{i} z_{30}}{D^{\prime}}=\frac{1.22 \lambda_{i}}{N . A .} \sim 1.22 \lambda_{i} \approx 0.5 \mu \mathrm{m}$. Here, the N.A. (Numerical Aperture) of holographic lens is close to 1 and the recording wavelength $\lambda_{\mathrm{i}}$ is $405 \mathrm{~nm}$. It is this diffraction limit that determines the minimum $d$ of a photodetector to be used and, therefore, the minimum $\Delta z$ for starlight collection.

According to the geometry shown in Fig. 7a, the amount of light collected over a diameter $d(\equiv \Delta y)$ is equivalent to those light distributed over a length $\Delta z$ along the $z$ axis. If the light incident angle is $\theta$, we have $\Delta z=\frac{\Delta y}{2 \times \tan \theta}$. Here, $\theta=\tan ^{-1}\left(\frac{\mathrm{R}}{z_{30}}\right)=\tan ^{-1}\left(\frac{20}{388}\right)=3.0^{\circ}$. We may now re-examine the experimental configuration and spectral linewidth shown in Fig. 6a,b, respectively. Since the fiber has a diameter $d=\Delta y=400 \mu \mathrm{m}$, the corresponding detecting length $\Delta z=\frac{8 y}{2 \times \tan \theta}=\frac{400 \times 10^{-3}}{2 \times 0.05}=4 \mathrm{~mm}$. By Eq. (5), the predicted spectral linewidth $|\Delta \lambda| \cong \Delta z \times 1.33 \times 10^{-3} \mathrm{~nm} / \mu \mathrm{m} \cong 5.3 \mathrm{~nm}$ at $z_{30}=388 \mathrm{~mm}$. This finding agrees with the measured results shown in Fig. 6 b within $\sim 2 \mathrm{~nm}$.

Separately, the Fresnel hologram may be utilized for a sensitive starlight detection. We will estimate the minimum detectable power of a micro-PMT detector and then examine the star light flux of alpha Lyrae, the 5th brightest star in the night sky. A typical micro-PMT has a noise level $\mathrm{V}_{\text {noise }}=6 \mathrm{mV}$ for a detector area 


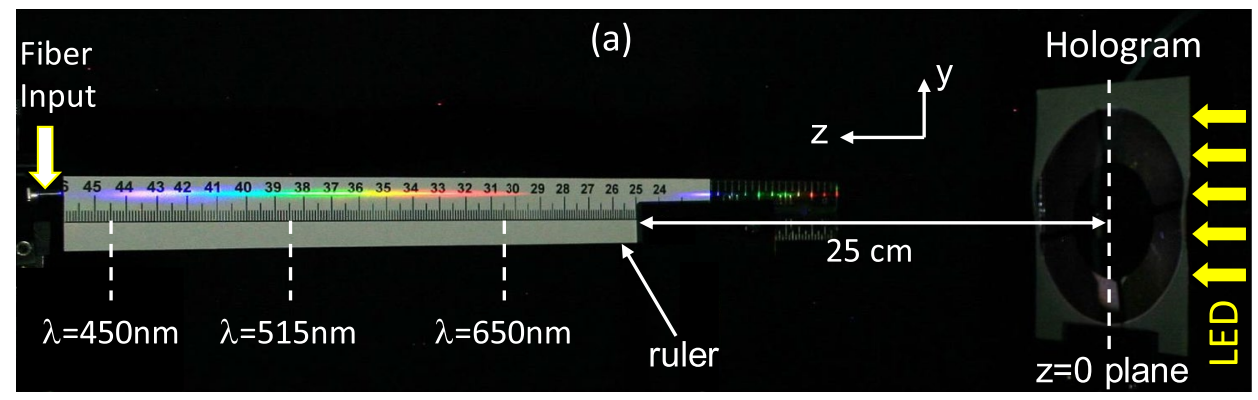

(b)

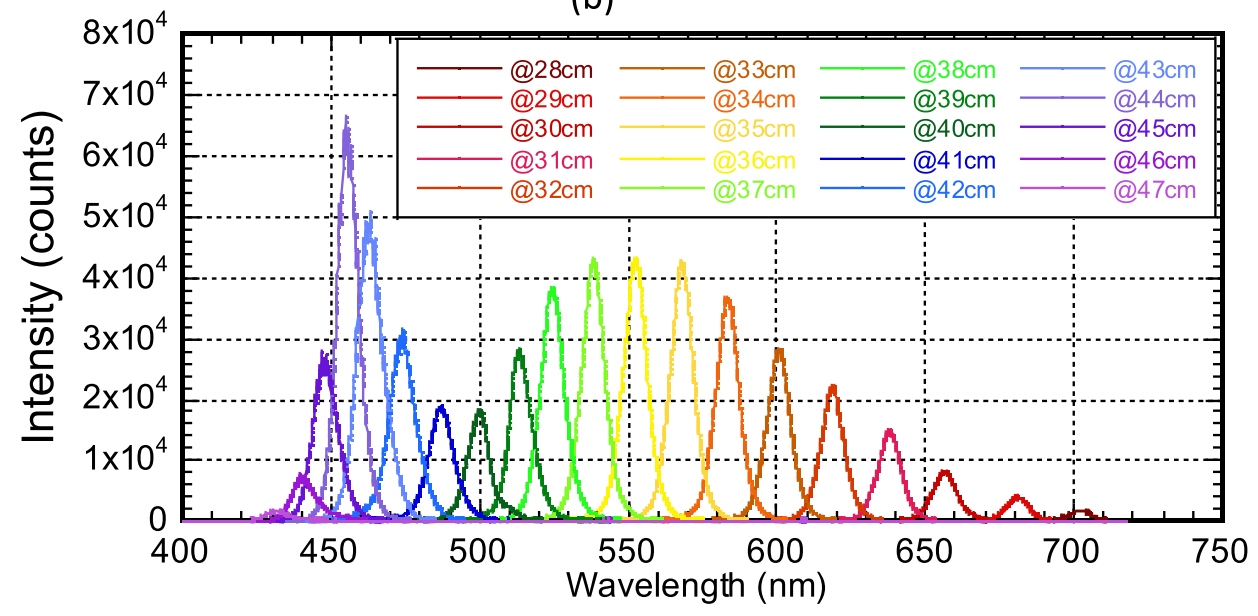

Figure 6. Reconstructed image and spectroscopic measurement of the Fresnel hologram. (a) A photo of the reconstructed image using a collimated LED white light. The ruler is placed near the optical axis, $\mathrm{z}$-axis, to illustrate the chromatic behavior. (b) A series of spectral curves taken at different positions along the optical axis using an optical fiber of core diameter $\mathrm{d}=400 \mu \mathrm{m}$.

$\mathrm{A}=3 \times 3 \mathrm{~mm}^{218}$. Its detectivity is such that a $1 \mathrm{pW}$ will lead to a signal level of $\mathrm{V}_{\text {signal }}=15 \mathrm{mV}$. This gives a signalto-noise ratio of $15 \mathrm{mV} / 6 \mathrm{mV}=2.5$. Let us assume that this is an acceptable $\mathrm{S} / \mathrm{N}$ ratio and $1 \mathrm{pW}$ is the minimum detectable power of such a PMT detector. For our hologram detecting system, incoming light may be concentrated to $\mathrm{A}_{\mathrm{det}} \sim 500 \times 500 \mu \mathrm{m}^{2}$, and so the PMT detector size may be reduced accordingly. Given that a detector's noise voltage, such as the Johnson and shot noises, is proportional to the square root of the optically active area ${ }^{19}$, its noise voltage becomes $\mathrm{V}^{\prime}{ }_{\text {noise }}=1 \mathrm{mV}$ when its area is reduced by a factor 36 times to $A_{\text {det }} \sim 500 \times 500 \mu \mathrm{m}^{2}$. So, the minimum detectable voltage is also reduced and given by $\mathrm{V}_{\text {signal }}^{\prime}=\mathrm{V}_{\text {noise }}^{\prime} \times 2.5=2.5 \mathrm{mV}$. The minimum detectable power of a reduced area micro-PMT is $1 \mathrm{pW} / \sqrt{36} \approx 1.6 \times 10^{-13} \mathrm{~W}$. If we further assume that our $10 \times 10 \mathrm{~cm}^{2}$ Fresnel hologram as a $30 \%$ diffraction efficiency, the minimum detectable power of a reduced area $\mathrm{PMT}$ is $\mathrm{P}_{\min } \approx 5.3 \times 10^{-13} \mathrm{~W}$.

We now examine the star light flux. A star with apparent magnitude $\mathrm{V}=0$ and zero color which is approximately the apparent magnitude and color of alpha Lyrae, the 5th brightest star in the night sky, has a flux of $37.4 \times 10^{-12} \mathrm{~W} / \mathrm{m}^{2} / \mathrm{nm}^{20,21}$ over the wavelength range of the V filter. The V filter has a spectral width of $88 \mathrm{~nm}$ and is centered at $\lambda=545 \mathrm{~nm}$. Therefore, for a hologram that is $10 \times 10 \mathrm{~cm}^{2}$, the flux of a 0 th magnitude stars with zero color is $37.4 \times 10^{-14} \mathrm{~W} / \mathrm{nm}$. From Eq. (5), the spectral width for a detector size of $d=500 \mu \mathrm{m}$ and $\Delta z=5 \mathrm{~mm}$ is $\Delta \lambda=6.65 \mathrm{~nm}$. So, the starlight power is $\left(37.4 \times 10^{-14} \mathrm{~W} / \mathrm{nm}\right) \times(6.65 \mathrm{~nm})=2.48 \times 10^{-12} \mathrm{~W}$. It is 4.5 times larger than the minimum detectable power of our Fresnel hologram with $30 \%$ diffraction efficiency. As a result, the Fresnel hologram holds great promise for a sensitive detection for space exoplanet telescope.

\section{Conclusion}

In summary, we present the design of an unique annular Fresnel hologram based on the interference of two spherical waves. Through an analytical design, we found that the focal-length of our hologram can be tailored by tuning the radius-of-curvature of the two spherical waves. The grating period of the Fresnel hologram decreases monotonically from the center to the outer region and brings the incoming star-light to focus. Experimentally, we have successfully recorded our Fresnel hologram based on spherically symmetrical waves, with a focal-length by design. Furthermore, we have demonstrated a large on-axis dispersive behavior of an incoming, collimated light wave. This work represents the first successful implementation of a scale model optical system for realizing a telescope Fresnel hologram, which can achieve a spectral dispersion of $\lambda=440-705 \mathrm{~nm}$ over an on-axis 

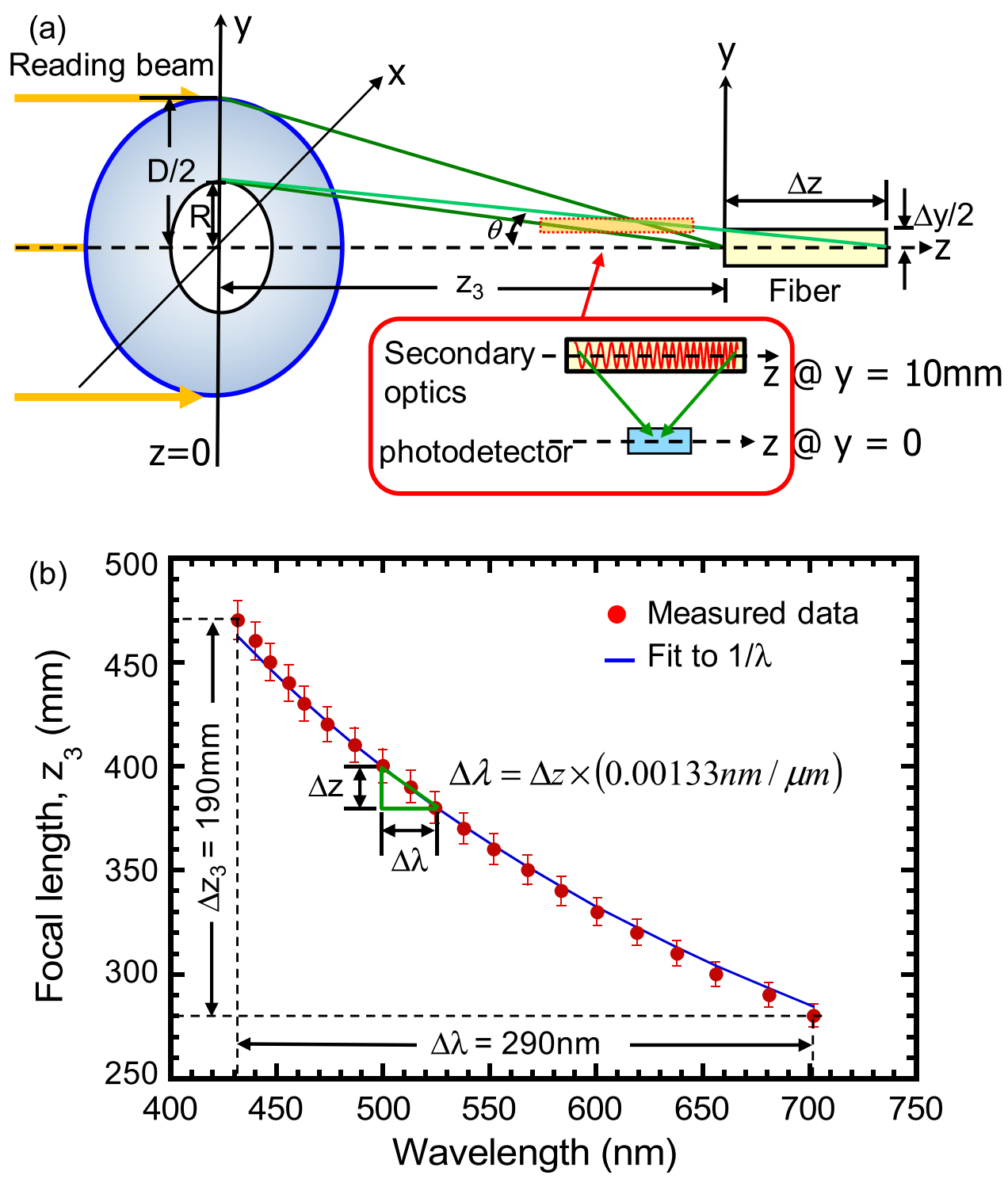

Figure 7. Spectra dispersion and resolution of Fresnel Hologram on the optical axis. (a) Reading geometry of the hologram and the image of a monochromatic light $\lambda$ at its focal length $z_{3}$. The detecting length $\Delta z$ and width $\Delta y$ are also indicated. (b) A summary plot of the measured focal length $z_{3}$ versus reading $\lambda$. The red dots are experimental data with $2 \%$ error bar and the blue curve is a simulated result $\left(z_{\mathrm{O}}=-25.2 \mathrm{~cm}, z_{\mathrm{R}}=-72 \mathrm{~cm}\right)$.

distance of $190 \mathrm{~mm}$. Therefore, our proposed DUET can simultaneously perform high resolution spectroscopy, high detection sensitivity and have a low areal mass for space exploration.

\section{Methods}

Reconstruction of the on-axis hologram with two spherical recording waves. Let us consider a plane wave $U_{\mathrm{p}}$ illuminating the hologram at normal incidence and with a wavelength $\lambda$, Fig. $2 \mathrm{a}$. The hologram is recorded using two spherical waves of wavelength $\lambda_{\mathrm{i}}$, placed at $z_{\mathrm{R}}$ and $z_{\mathrm{O}}$, respectively. The recorded, constructive interference regions attenuate the illuminating (reading) light, so the transmittance of the hologram ${ }^{22,23}$ is:

$$
t_{A}(x, y, z=0)=t_{0}-\kappa \times I(x, y, z=0)
$$

Here, $t_{0}$ and $\kappa$ are both constants and their values dependent on the holographic material chosen. Accordingly, the transmitted wave propagating through the hologram, $U_{t}(x, y, z=0)$, is given by: 


$$
\begin{aligned}
U_{t}(x, y, z=0) & =U_{P} \times t_{A}(x, y, z=0)=U_{P} \times\left[t_{0}-\kappa \times I(x, y)\right] \\
& =U_{P} \times\left[t_{0}-\kappa\left|U_{R}+U_{O}\right|^{2}\right] \\
& =U_{P}\left(t_{0}-\kappa\left|U_{R}\right|^{2}\right)-\kappa U_{P}\left|U_{O}\right|^{2}-\kappa U_{P} U_{R}^{*} U_{O}-\kappa U_{P} U_{R} U_{O}^{*} \\
& =U_{1}+U_{2}+U_{3}+U_{4}
\end{aligned}
$$

Here, $U_{1}$ and $U_{2}$ are both constants of $(x, y)$. They are plane waves that directly transmit through the hologram. $U_{3}$ and $U_{4}$ are complex conjugate terms. They represent the two diffracted spherical waves. $U_{3}$ is a real image and $U_{4}$ a virtual image. $U_{3}$ may be further computed to be:

$$
\begin{gathered}
U_{3}(x, y, z=0)=-\kappa U_{P} U_{R}^{*} U_{O}=-\kappa A_{P} A_{R}^{*} A_{O} e^{i\left(\phi_{A}+\phi_{O}-\phi_{R}\right)}=A_{3} e^{i \phi_{3}} \\
\phi_{3} \equiv \phi_{P}+\phi_{O}-\phi_{R}=-\frac{\pi}{\lambda}\left[\left(x^{2}+y^{2}\right)\left(\frac{1}{z_{O}} \frac{\lambda}{\lambda_{i}}-\frac{1}{z_{R}} \frac{\lambda}{\lambda_{i}}\right)-2 x\left(\frac{x_{O}}{z_{O}} \frac{\lambda}{\lambda_{i}}-\frac{x_{R}}{z_{R}} \frac{\lambda}{\lambda_{i}}\right)-2 y\left(\frac{y_{O}}{z_{O}} \frac{\lambda}{\lambda_{i}}-\frac{y_{R}}{z_{R}} \frac{\lambda}{\lambda_{i}}\right)\right]
\end{gathered}
$$

When $\left(x_{\mathrm{R}}, y_{\mathrm{R}}\right)=(0,0)$ and $\left(x_{\mathrm{O}}, y_{\mathrm{O}}\right)=(0,0), \phi_{3}$ can be simplified as:

$$
\therefore \phi_{3} \equiv \phi_{P}+\phi_{O}-\phi_{R}=-\frac{\pi}{\lambda}\left[\left(x^{2}+y^{2}\right)\left(\frac{1}{z_{O}} \frac{\lambda}{\lambda_{i}}-\frac{1}{z_{R}} \frac{\lambda}{\lambda_{i}}\right)\right]
$$

Let us demand that the wave represented by $U_{3}$ is to produce a point image at $z>0$, so it must be a spherical wave originated from a point source at $\left(x_{3}, y_{3}, z_{3}\right)$. Similar to Eq. (2), $U_{3}$ may also be written as:

$$
\begin{aligned}
U_{3} & =\left(A_{3} / z_{3}\right) e^{i k \sqrt{\left(x-x_{3}\right)^{2}+\left(y-y_{3}\right)^{2}+\left(z-z_{3}\right)^{2}}} \approx\left(A_{3} / z_{3}\right) e^{i k \phi_{3}} \\
\phi_{3} & \equiv-\frac{\pi}{\lambda z_{3}}\left(x^{2}+y^{2}-2 x x_{3}-2 y y_{3}\right)
\end{aligned}
$$

For the first order diffraction, we match and equate the phase factors $\phi_{3}$, between Eqs. (9) and (10). As a result, the focal point $\left(x_{3}, y_{3}, z_{3}\right)$ of the image is given by:

$$
\begin{aligned}
& x_{3}=y_{3}=0 \\
& \frac{1}{z_{3}}=\left(\frac{1}{z_{R}}-\frac{1}{z_{O}}\right) \frac{\lambda}{\lambda_{i}} \text { or } z_{3} \lambda=\left(\frac{1}{z_{R}}-\frac{1}{z_{O}}\right)^{-1} \lambda_{i}
\end{aligned}
$$

Received: 22 July 2021; Accepted: 28 September 2021

Published online: 21 October 2021

\section{References}

1. Lin, S. Y., Chow, E., Hietala, V., Villeneuve, P. R. \& Joannopoulos, J. D. Experimental demonstration of guiding and bending of electromagnetic waves in a photonic crystal. Science 282, 274 (1998).

2. Lin, S. Y., Hietala, V. M., Wang, L. \& Jones, E. D. A highly dispersive photonic band gap prism. Opt. Lett. 21, 1771 (1996).

3. Hsieh, M. L., Chen, H. Y., Peng, C. T. \& Lin, S.-Y. Lens-less bending and concentration of light by volume hologram. Opt. Commun. 365, 12-16 (2015).

4. Hsieh, M. L., Chen, H. Y. \& Lin, S.-Y. Observation of a large diffraction efficiency and efficiency enhancement of PQ/PMMA materials. Opt. Commun. 308, 121-124 (2014).

5. Barton, M. et al. Fabrication of large-aperture lightweight diffractive lenses for use in space. Appl. Opt. 40, 447 (2001).

6. Meinel, A. B. \& Meinel, M. P. Parametric dependencies of high-diffraction-order achromatized aplanatic configurations that employ circular or crossed-linear diffractive optical elements. Appl. Opt. 41, 7155 (2002).

7. Meinel, A. B. \& Meinel, M. P. Large membrane space optics: Imagery and aberrations of diffractive and holographic achromatized optical elements of high diffraction order. Opt. Eng. 41, 1995 (2002).

8. MacEwen, H. A. \& Breckinridge, J. B. Large diffractive/refractive apertures for space and airborne telescopes. Proc. SPIE Sens. Syst. Space Appl. 8739, 873904 (2013).

9. Andersen, G. Large optical photon sieve. Opt. Lett. 30(22), 2976-2978 (2005).

10. Ditto, T. D., Friedman, J. F. \& Content, D. A. Astronomical telescope with holographic primary objective. Proc. SPIE 8146, 814615 (2011).

11. Ditto, T. D. et al. Optical space telescope without mirrors. Proc. SPIE 11443, 114431B (2020).

12. Sweatt, W. C. Describing holographic optical elements as lenses. J. Opt. Soc. Am. 67(6), 803 (1977).

13. Benton, S. A. \& Bove, V. M. Holographic Imaging, Chap. 9 (Wiley, 2008) ISBN: 978-0-470-22412-0.

14. Bennett, C. A. Principle of Physical Optics, Chap. 4, 125, Chap. 6, 321, Chap. 6, 295 (Wiley, 2008).

15. Zemax 13 is a commercially available optical design program from Radiant Zemax LLC 1990-2013. https://www.radiantzemax. com.

16. A description of the Cassegrain Reflecting telescope. https://en.wikipedia.org/wiki/Reflecting_telescope.

17. Schubert, E. F. Light Emitting Diodes 1st edn, 251 (Cambridge University Press, 2003).

18. Hamamatsu's product on "Micro-PMT photodetector". https://www.hamamatsu.com/us/en/product/optical-sensors/pmt/micro$\mathrm{pmt} / \mathrm{micro}$-pmt-package/index.html.

19. Dereniak, E. L. \& Boreman, G. D. Infrared Detectors and Systems 168-174 (Wiley, 1997).

20. Bessell, M. S. Spectrophotometry: Revised standards and techniques. Pub. Astron. Soc. Pac. 111, 1426-1433 (1999).

21. Bessell, M. S. Standard photometric systems. Annu. Rev. Astron. Astrophys. 43, 293-336 (2005).

22. Collier, R. J., Burckhardt, C. B. \& Lin, L. H. Optical Holography 72 (Academic Press, 1971) (Chap. 3, Section 3.31 “In-line Hologram Images").

23. Kogelnik, H. Coupled wave theory for thick hologram gratings. Bell Syst. Tech. J. 48(9), 2909-2947 (1969). 


\section{Acknowledgements}

MLH and TDD gratefully acknowledge the financial support from NASA Innovative Advanced Concepts Phase I Study under Grant for Research 80NSSC19K0967NASA. YWL and SYL acknowledge partial support from Taiwan's Department of Education (DOE) through a short-term exchange Yushan scholar program.

\section{Author contributions}

M.L.H.: provide theoretical input and formulate theoretical framework, implement optical setup, perform optical testing and experimental data analysis; T.D.D.: design and formulate the Fresnel hologram for telescope; Y.W.L.: perform optical testing and experimental data analysis; S.-H.L.: perform optical testing; H.J.N.: provide input and estimation of star light detection for space exoplanet telescope; S.Y.L.: design the experiment and perform optical testing, data analysis, draft the manuscript.

\section{Competing interests}

The authors declare no competing interests.

\section{Additional information}

Correspondence and requests for materials should be addressed to S.-Y.L.

Reprints and permissions information is available at www.nature.com/reprints.

Publisher's note Springer Nature remains neutral with regard to jurisdictional claims in published maps and institutional affiliations.

(c) (i) Open Access This article is licensed under a Creative Commons Attribution 4.0 International License, which permits use, sharing, adaptation, distribution and reproduction in any medium or format, as long as you give appropriate credit to the original author(s) and the source, provide a link to the Creative Commons licence, and indicate if changes were made. The images or other third party material in this article are included in the article's Creative Commons licence, unless indicated otherwise in a credit line to the material. If material is not included in the article's Creative Commons licence and your intended use is not permitted by statutory regulation or exceeds the permitted use, you will need to obtain permission directly from the copyright holder. To view a copy of this licence, visit http://creativecommons.org/licenses/by/4.0/.

(C) The Author(s) 2021 